\title{
Spatiotemporal molecular approach of in utero electroporation to functionally decipher endophenotypes in neurodevelopmental disorders
}

\section{Sharon Margriet Kolk*, Annetrude Johanne de Mooij-Malsen and Gerard Julianus Maria Martens}

Department of Molecular Animal Physiology, Donders Institute for Brain, Cognition, and Behaviour, Nijmegen Centre for Molecular Life Sciences, Radboud University Nijmegen, Nijmegen, Netherlands

Edited by:

Alistair N. Garratt, Max Delbrück Center for Molecular Medicine, Germany

Reviewed by:

Josef Kittler, University College London, UK

Patrick Chappell, Oregon State

University, USA

${ }^{*}$ Correspondence:

Sharon Margriet Kolk, Department of Molecular Animal Physiology,

Donders Institute for Brain, Cognition, and Behaviour, Nijmegen Centre for Molecular Life Sciences, Radboud

University Nijmegen, Geert

Grooteplein Zuid 28, 6525 GA

Nijmegen, Netherlands.

e-mail:s.kolk@ncm/s.ru.nl
We have only just begun to decipher the complexity of our brain, including its maturation. Correct brain development and communication among brain areas are crucial for proper cognitive behavior. Brain area-specific genes expressed within a particular time window direct neurodevelopmental events such as proliferation, migration, axon guidance, dendritic arborization, and synaptogenesis. These genes can pose as susceptibility factors in neurodevelopmental disorders eventually resulting in area-specific cognitive deficits. Therefore, in utero electroporation (IUE)-mediated gene transfer can aid in creating valuable animal models in which the regionality and time of expression can be restricted for the targeted gene(s). Moreover, through the use of cell-type-specific molecular constructs, expression can be altered in a particular neuronal subset within a distinct area such that we are now able to causally link the function of that gene in that brain region to the etiology of the disorder. Thus, IUE-mediated gene transfer is an attractive molecular technique to spatiotemporally address the developmental aspects of gene function in relation to neurodevelopmental disorder-associated endophenotypes.

Keywords: neurodevelopment, in utero electroporation, animal model, gene transfer, migration, axon guidance
Our complex adult brain is able to make the daily connections between areas to present appropriate behavior in response to our environment. The higher cognitive functions include perception, attention, memory, problem solving, and emotion, and involves appropriate cross-talk between neuronal subpopulations in various brain regions. The development of these brain areas is a multi-step process that includes proliferation, migration, axon guidance, dendritic arborization, and synaptogenesis to establish the correct maturation of the areas and their connectivity. The various steps in brain area development are guided by a plethora of molecules that are expressed at a particular place at a specific time. To decipher the exact developmental mechanisms that underlie the etiology of neurodevelopmental disorders, it is necessary to unravel the function of these molecules in four dimensions.

Linkage and association studies have classified multiple candidate genes that might contribute to the clinical features of neurodevelopmental disorders. Many of them seem to be involved in the neurodevelopmental events of brain maturation. Their exact functions over time and in relation to other genes, however, remain elusive. Animal models provide compelling insight into cognitive endophenotypes of these disorders by unraveling the underlying biological mechanism of the disease (Gould and Gottesman, 2006; Kellendonk et al., 2009). By introducing molecular fluorescent control and gene target constructs, we are now able to monitor the mechanisms of (mis)development. Traditionally, the gene of interest would be molecularly targeted by homologous recombination, resulting in a systemic absence of the gene in the animal. However, besides a high level of variability in the knockout and transgenic lines, often molecular compensation mechanisms occur. Further, the molecular alteration in the complete organism shows low construct validity in these complex disorders and even in a Cre-Lox system a large part of the brain is targeted. For this reason, the innovative and exciting technique of in utero electroporation (IUE)-mediated gene transfer is a huge step forward in the research of biological functions of genes over time, especially in the study of psychiatric disorders (Fukuchi-Shimogori and Grove, 2001; Inoue and Krumlauf, 2001; Saito and Nakatsuji, 2001). Major advantages are the spatial and temporal restriction of gene introduction and the possibility to target more than one gene suitably at different developmental time points. Various constructs, either up- or downregulating the gene of interest, can be microinjected into the lateral ventricles of the developing embryonic brain. The construct is then incorporated into the ventricular surface by electroporation using a series of unipolar square wave pulses, driving expression into specific brain areas in which the position of the positive electrode defines the site of DNA entry (Figure 1A). For example, when the positive pole of the electrodes is positioned dorsally of the embryonic forebrain, the electroporated area exist of the more dorsofrontal brain areas such as the hippocampus (Figures 1A,B,, $\mathbf{B}^{\prime}$ ) and prefrontal cortex (PFC; Figures 1A,C, $\mathbf{C}^{\prime}$ ). More ventrolateral positioning of the positive electrode can specifically electroporate striatal aspects (Figures 1A,D, $\mathbf{D}^{\prime}$ ). By leaving the embryos undisturbed during further development, the timing of examination can be carefully chosen depending on the question asked. In contrast 


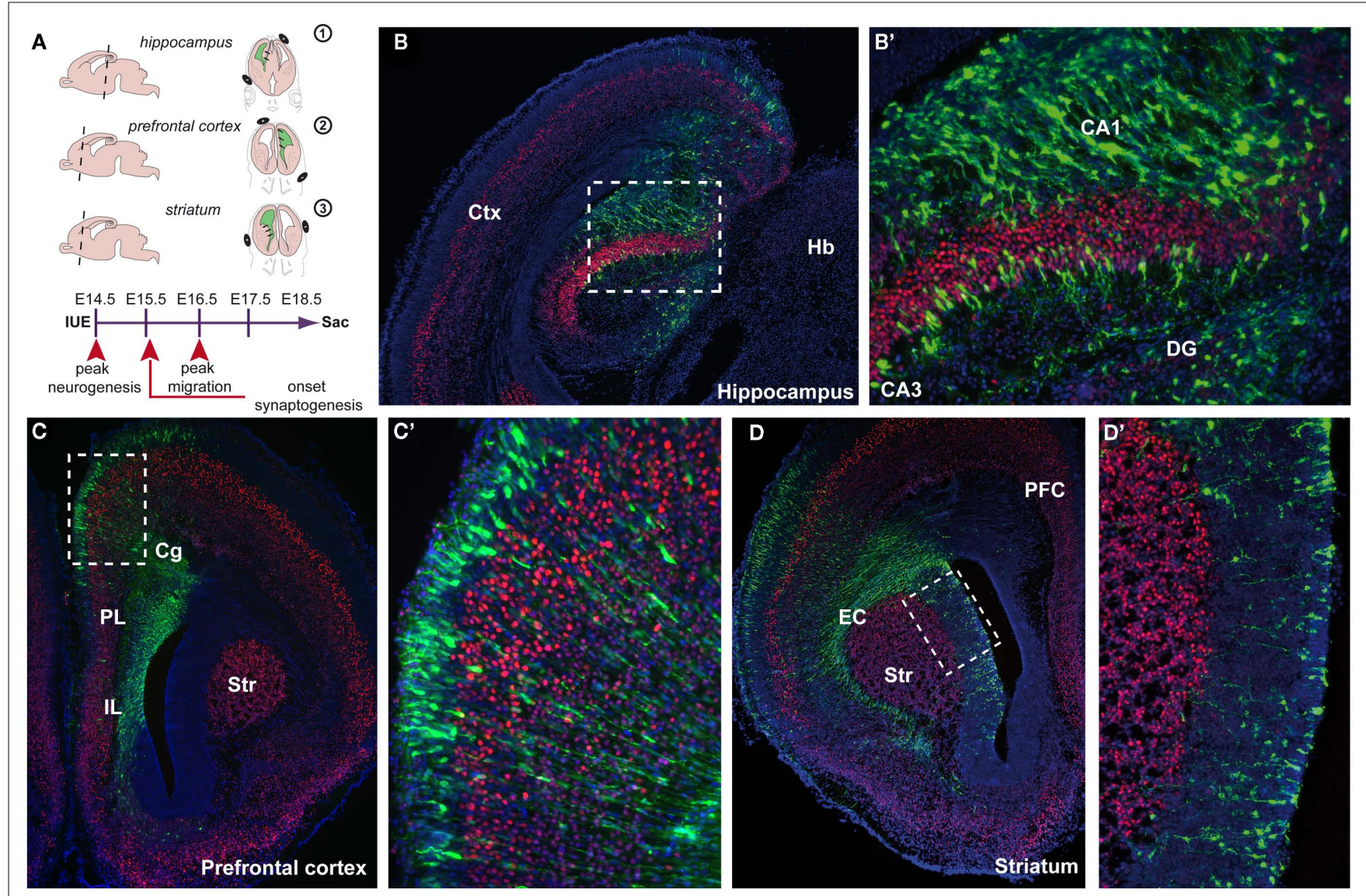

FIGURE 1 | Spatial confinement to areas involved in neurodevelopmental disorders using in utero electroporation (IUE) -mediated gene transfer. (A) Schematic representation of IUE into three areas (1) the hippocampus, (2) the prefrontal cortex, (3) the striatum. On the left side, a sagittal view is shown indicating the level of the coronal section (dotted line) shown on the right side where the position of the + and poles is indicated. IUE is performed at embryonic day 14.5 (E14.5) and embryos are left undisturbed for 4 days in utero (diu) before sacrifice (sac) at E18.5. Arrowheads indicate various neurodevelopmental events. (B) E18.5 coronal cryosection of an electroporated mouse brain (E18.5-4diu). Control construct CMV-YFP-N1 (green) is electroporated into the hippocampal anlage enlarged in (B') (boxed area), counterstained with the layer $V$ marker Ctip2 (red) and fluorescent Nissl (blue). (C) Electroporation was performed into the whole span of the medial prefrontal cortex (mPFC) including the infralimbic (IL), prelimbic (PL), and cingulate cortex (Cg). Boxed area is enlarged in (C'). (D) Electroporation was performed into the ventricular zone (VZ) of the caudate putamen (CP) close to the external capsule (EC). Boxed area is enlarged in $\left(\mathbf{D}^{\prime}\right)$. CA1, cornu ammonis 1; CA3, cornu ammonis $3 ; \mathrm{Cg}$, cingulate cortex; Ctx, cortex; DG, dentate gyrus; EC, external capsule; $\mathrm{Hb}$, habenula; Str, striatum. to the time-consuming generation of transgenic or mutant mouse lines, this method allows for quick analyses of the function of genes in a spatiotemporal manner.

The first major improvement of the technique is the spatial restriction that can be achieved within the developing brain (Figure 1). Different areas can be aimed for, depending on the position and/or angle of the positive pole of the electrical square wave current applied (Figure 1A). This is an improvement to nonaided in vivo viral infections where the infected area is usually large and dependent on the titer (Cetin et al., 2006). Areas known to be involved in neurodevelopmental disorders such as the hippocampus (Nakahira and Yuasa, 2005; Navarro-Quiroga et al., 2007), PFC, and the striatum can now be reached individually and electroporated with the gene construct(s) of interest (Figures $\mathbf{1 A}-\mathbf{D}^{\prime}$ ). In this way, the brain region of interest is affected without disturbing the rest of the embryo. By precisely knocking down a specific gene in only the PFC during development, behavioral abnormalities can be screened postnatally and causal connections can be made with certain endophenotypes that involve working memory or information processing (Gould and Gottesman, 2006; Niwa et al., 2010). For example, knocking down DISC1, a genetic susceptibility factor in schizophrenia, in the PFC using IUE will lead to particular behavioral deficits in the adult animal (Niwa et al., 2010). By targeting the ganglionic eminence or preoptic area, populations of interneurons can be genetically manipulated (Borrell et al., 2005; Lopez-Bendito et al., 2008; Gelman et al., 2009). By specifically labeling sets of interneurons by IUE and manipulating GABAergic circuit formation is a big step forward in neuropsychiatric research as interneurons control the function of cortical networks implicated to be involved in the etiology of neurodevelopmental disorders (Fazzari et al., 2010). The dimension of the transfected area can be adjusted 
by fine-tuning the diameter of the paddle-type electrodes, by using wire-type electrodes and by altering the time between the micro-injection of the DNA and the application of the series of currents. The developmental time point of the micro-injection extends the range of brain areas to be targeted. The retina, for example, can be reached by early micro-injection, with or without facilitation by ultrasound guidance (Garcia-Frigola et al., 2007; Punzo and Cepko, 2008; Petros et al., 2009a; Punzo et al., 2009). Thus, any brain (sub)area can currently be targeted, including areas more difficult to reach such as the midbrain (Alvarez-Maya et al., 2001; Willett and Greene, 2011), dorsal thalamus (Bonnin et al., 2007), hypothalamus (Gelman et al., 2009), amygdala (Remedios et al., 2007; Soma et al., 2009), or rhombic lip/cerebellum (Kawauchi et al., 2006), by manipulating the timing of introduction, the position of the injection and the position of the electrodes.

By manipulating the timing of introduction of the construct of interest (either overexpression or knockdown), various developmental events can be addressed such as proliferation, migration (both radial as well as tangential), axon guidance, dendritic maturation, and synaptogenesis. One of the first studies that used IUE to examine early patterning of the cerebral cortex by Fgf8 was by the lab of Grove (Fukuchi-Shimogori and Grove, 2001). Regional patterning was changed such that overexpression of Fgf8 by IUE shifted the position of the somatosensory cortex more caudal, including occasionally the whole barrel field. When progenitor cells are electroporated with plasmids directing the overexpression or knockdown of a target gene, proliferation can be affected which can be tested in vivo in combination with the use of BrdU which incorporates into the S-phase of dividing cells (Figures 2A,B). For instance, when the forward signaling of the receptor tyrosine kinase EphA4 is diminished in cortical progenitors through IUE by introducing a dominant-negative EphA4 construct lacking the kinase domain, cell division is affected (North et al., 2009). Another developmental event that can be studied through the use of IUE is neuronal migration (Figures 2C-F). This is particularly apparent in the "inside-out" layering of the cerebral cortex where late-born neurons migrate to their more superficial positions past the early born neurons in the deeper layers. Defects in migration can thus result in aberrant layering or even heterotopias (Kawauchi et al., 2003; Bai et al., 2008). Using layerspecific markers along with BrdU to "birthdate" cells in combination with IUE, defects in migration can be easily detected (Figures 2D-F). Early axon guidance events can be altered by either molecularly changing the target area or the area where the projections originate from (Figures 2G,H). Knocking down the levels of Disc1 in a specific subset of pyramidal neurons in the developing mouse PFC actually diminishes the dopaminergic mesocortical projections leading to prominent behavioral problems in adulthood (Niwa et al., 2010). Also it was shown that ectopic EphB1 expression in a subset of retinal ganglion cells established using IUE convincingly altered their projection trajectory (Petros et al., 2009b). Also, the later events in development such as Fgf8 dendritic arborization and connectivity can be studied using IUE (Figures 2I,J). The molecular mechanisms underlying late neurodevelopmental events such as synaptogenesis and synapse maturation can be examined by introducing the plasmid early and analyzing the brain areas of interest postnatally (Elias et al., 2008). Furthermore, a developmentally very late electroporation paradigm (at E18.5 or postnatal) can be performed and will drive expression in mainly the astrocytic progenitors (LoTurco et al., 2009).

To manipulate gene expression, constructs can be designed to target specific cells at a specific time (Miyagi et al., 2004; Gal et al., 2006; Wang et al., 2007b). Experiments using IUE can be performed using either knockdown by RNA interference (RNAi), antisense morpholinos, dominant-negative (DN; North et al., 2009) constructs, or toxins (Wang et al., 2007a) to manipulate cell signaling, or a Cre-Lox system to fine-tune expression time or combinations of constructs in which a full-length (FL) version is later used to rescue a particular phenotype (Mizutani and Saito, 2005; Matsuda and Cepko, 2007). The high capacity and efficiency of viral vectors makes them suitable for the overexpression of large full-length constructs but can however elicit immune responses in the adult brain (Davidson and Breakefield, 2003; Cetin et al., 2006). The downside of non-viral vectors on the other hand is that they are expected to be preserved episomally and thus not suitable for lineage trace experiments. Promoters such as the cytomegalovirus (CMV) and elongation factor $1 \alpha$ $(\mathrm{EF} 1 \alpha)$ are not cell-type-specific and yield moderate expression levels that persist after birth and can therefore be used to study various developmental events (Figure 3). A battery of fluorescent elements can be either incorporated into the construct or co-electroporated with another construct to visualize the expression pattern (Figures 3A,B). Nowadays the most commonly used knockdown construct is the micro-RNA30-based short hairpin RNA (shRNA) for RNAi under the ubiquitous polymerase II promoter like chicken actin (CAG; Figure 3C). The knockdown of doublecortin-like (DCL) for example resulted in a reduced proliferation of neurons and radial glia fiber instability (Vreugdenhil et al., 2007). Distinct neurodevelopmental events in a subset of neurons within a particular region can now be controlled using cell-type-specific regulatory elements (Matsuda and Cepko, 2007; LoTurco et al., 2009; Manent et al., 2009). Cell-type-specific promoter or regulatory elements such as Thy1, Nestin, T $\alpha 1$, BLBP, PDGF, GLAST (Gal et al., 2006), CAMKII, Synapsin, DCX (Wang et al., 2007b), NSE but also ER81 and Ngn2 (Langevin et al., 2007) can drive expression of a fluorescently labeled construct into a particular neuronal cell population in which the time of expression can be both dependent on the time of electroporation and the period of expression of the regulatory element (Figure 3A). In this way endophenotypes can be further unraveled because of the specificity of the gene manipulation. To dissect gene-gene interaction, multiple constructs of different genes can be electroporated at the same time which is again a great step ahead in deciphering the molecular pathways underlying cognitive deficits associated with neurodevelopmental disorders (Kamiya, 2009). Neuronal migration is often affected in these disorders and Young-Pearse et al. (2007) showed that the effect on migration, by knocking down APP and Dab1, could be later recued using overexpression of Dabl. Likewise, when RNAi of Lis1 and dynein mutants where expressed at the same time their dual role in neuronal migration could be unraveled (Tsai et al., 2007). 

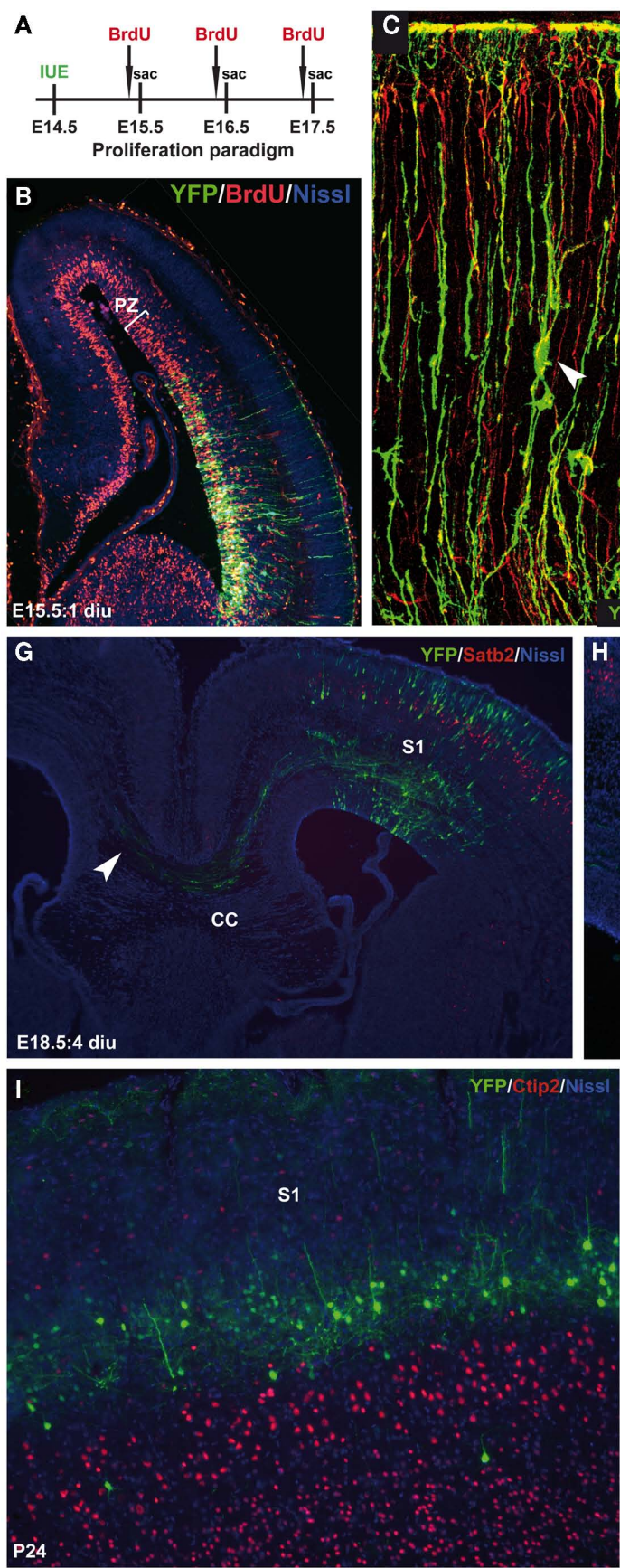
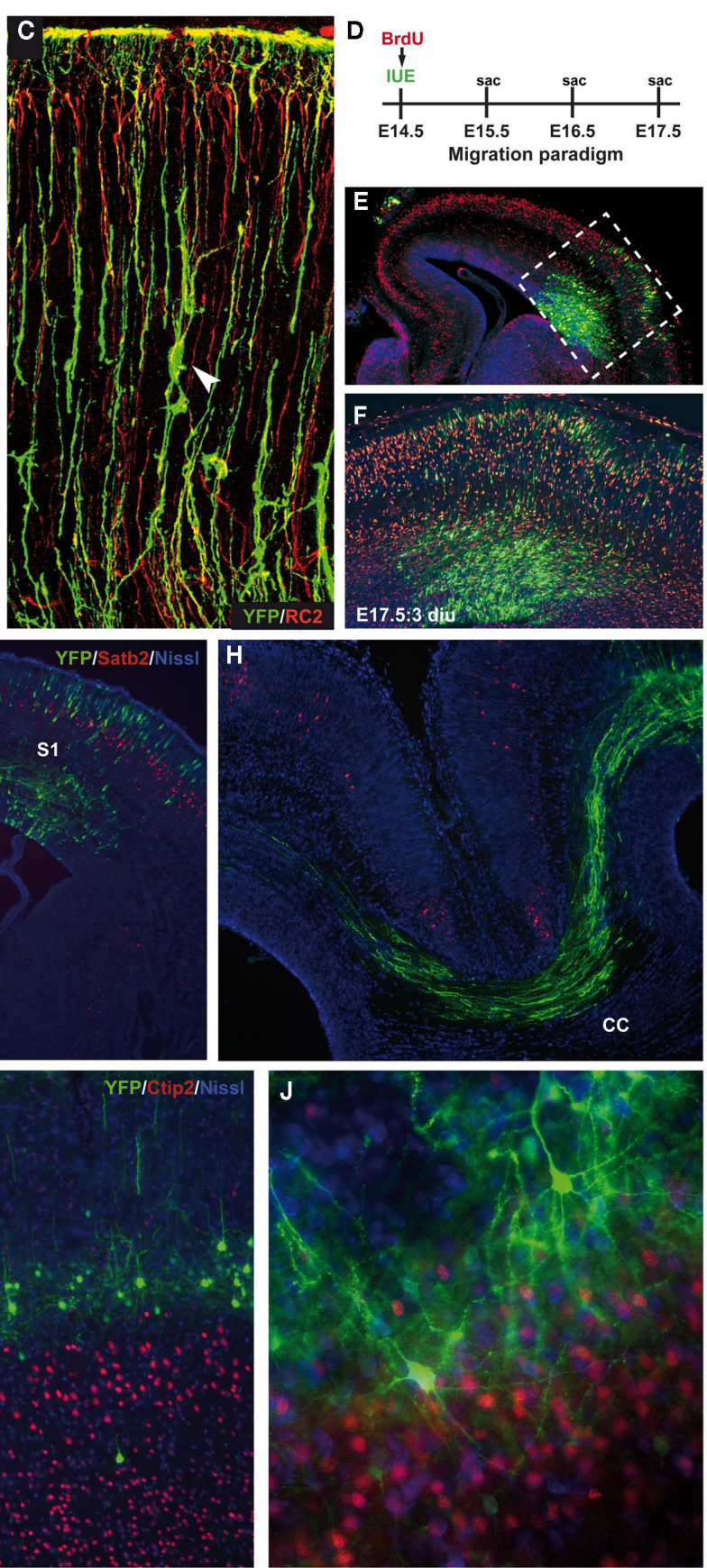

FIGURE 2 |Temporal restriction of gene introduction during various neurodevelopmental events. (A) Schematic representation of the proliferation paradigm using in utero electroporation (IUE). Two hours prior to sacrifice (sac), BrdU is injected to label dividing cells. (B) E15.5 coronal section showing the cerebral cortex with in red the BrdU-labeled cells within the proliferative zone (PZ) and in green the CMV-YFP-N1 construct (YFP), counterstained with fluorescent Nissl (blue) 1 day after IUE (1 diu). (C) Confocal image of YFP-labeled neurons and radial glia (green) co-stained with the radial glia marker RC2 (red). Arrowhead indicates a migrating neuron "climbing the robe" of a radial glia. (D) Schematic representation of the migration paradigm using IUE. Just after surgery BrdU is injected to label dividing cells and "birthdate" them. (E) E17.5 coronal section showing the cerebral cortex with BrdU-labeled cells (red) that have migrated to a certain position within the cortical plate (CP) combined with a YFP control construct (green), counterstained with fluorescent Nissl (blue) 3 days after IUE (3 diu). As soon as the YFP-positive neurons become post-mitotic they start to migrate and can be double labeled with BrdU (yellow). (F) Is an enlargement of the boxed area in (E). (G) E18.5 coronal section showing an YFP-labeled (green) somatosensory cortex (S1) projecting toward the other hemisphere through the corpus callosum (CC) counterstained with superficial layer marker Satb2 (red) and fluorescent Nissl (blue). (H) Is an enlargement of the projections through the CC in (G). (I) P24 coronal section showing

YFP-positive pyramidal neurons (green) in layer IV of the S1 on top of the layer $\checkmark$ marker Ctip2 (red). (J) Network formation of YFP-positive pyramidal neurons with extended dendritic branches and axonal projections. Counterstained with Ctip2 (red) en fluorescent Nissl (blue). 


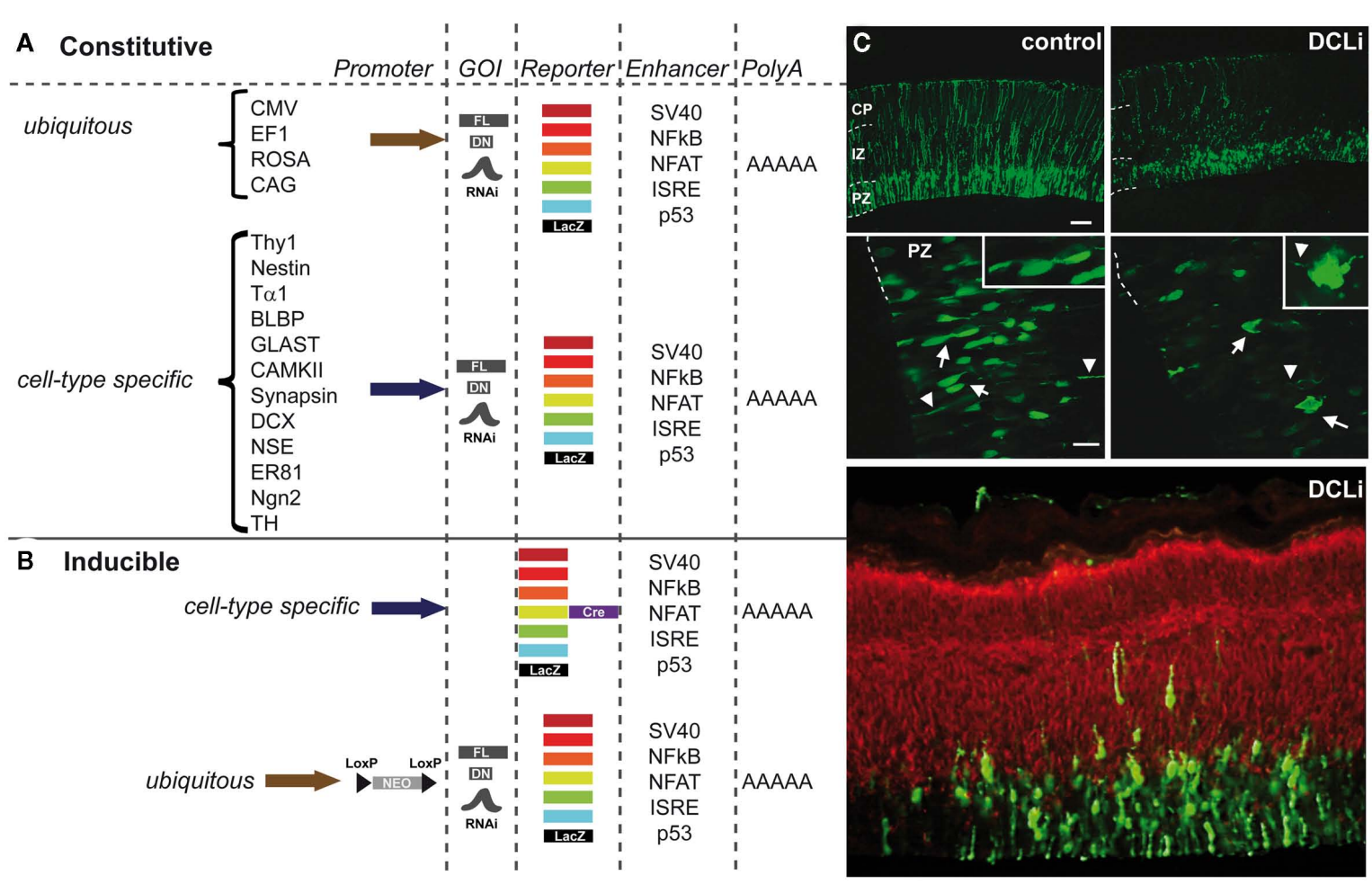

FIGURE 3 | Constructs for use in in utero electroporation (IUE) to direct expression in a four-dimensional way. (A) Schematic

representation of the most frequently used constructs that can be constitutively expressed under either a ubiquitous promoter (e.g., CMV, cytomegalovirus; EF1, elongation factor 1; ROSA, ROSA26 TATA-less promoter; CAG, chicken actin combined with CMV enhancer) or a cell-type-specific promoter (e.g., Thy1, THYmocyte differentiation antigen 1 driving expression in axons of neurons; Nestin, neuronal stem cell tubulin neuronal promoter; T $\alpha 1, \alpha$-tubulin neuronal promoter; BLBP, brain lipid-binding protein radial glial specific promoter; GLAST, glutamate-aspartate transporter radial glial/astrocytic promoter; CAMKII, calcium/calmodulin-dependent kinase II neuronal promoter specific for forebrain; Synapsin, neuronal promoter; DCX, doublecortin neuroblast-specific promoter; NSE, neuron-specific enolase drives expression in neurons and to some extent in glial cells; ER81, ETS family transcription factor driving expression in specific subset of neurons; Ngn2, neurogenin2 driving expression in neurons; $\mathrm{TH}$, tyrosine hydroxylase dopaminergic neuron-specific promoter). These promoters can drive expression of among others full-length (FL) constructs, dominant-negative (DN) constructs or, e.g., shRNAs of genes of interest (GOI) to establish knockdown via RNAi. The reporter element can be either a fluorescent protein ranging from far red, red, orange, yellow, green, or cyan fluorescence or LacZ. Often enhancer elements (e.g., SV40, NFkB, NFAT, ISRE, or p53) are used, but this is not always essential to drive the expression. Electroporation constructs are all terminated with a polyA tail. (B) Schematic representation of the commonly used inducible constructs such as the Cre-reporter construct expressed under an ubiquitous or a cell-type-specific promoter. The $\mathrm{GOI}$ containing construct can be under the control of an ubiquitous promoter (usually CAG) a neomycin (neo)-cassette that is floxed (loxP before and after) and thus excised upon addition of tamoxifen at a specific time point in development. (C) Example of a knockdown experiment using IUE with an off-target construct (control) and RNAi against the doublecortin-like gene (pSuper-DCL183 or DCLi). Both in the proliferative zone (PZ) as well as in the intermediate zone (IZ) and cortical plate (CP) neurons are reduced in number and the radial fibers are severely disrupted. Green is the construct, red is a $\beta$-tubulin staining (Tuj1). Adapted from Vreugdenhil et al. (2007).
Inducible models generated through the use of IUE have opened a new dimension to study the complexity of gene function(s) in the development of brain areas. The Cre-LoxP system can be used in combination with the ubiquitous CAG promoter or with above-mentioned regulatory elements that will allow expression in only a subset of neurons in a particular area and in a specific, carefully chosen time window through administration of tamoxifen inducing the Cre-ER(T) recombinases (Figure 3B). In this way, the onset of gene expression/suppression can be manipulated, for example at the peak of postnatal synaptogenesis avoiding the effect of this particular gene targeting on earlier events like neurogenesis or migration (Mizutani and Saito, 2005). Such an inducible system can furthermore be used in conjunction with the formerly performed effective RNAi-based knockdown where a possible rescue can be put in place postnatally (Matsuda and Cepko, 2007).

Together, IUE-mediated gene transfer has shown to be an exceptional powerful tool to create animal models in which both the spatial as well as the temporal aspects of gene expression can be manipulated. In future studies, adjustments like different sizes and shapes of the electrodes will further enhance the ability to specifically target a (sub)area. Inducible systems using chemical agents or optogenetic light-sensitive constructs such as channel-or halorhodopsin-assisted IUE (Petreanu et al., 2007; Lewis et al., 2009; Zhang et al., 2010) will further restrict the expression window in space and time, and thus a more 
precise control of gene manipulations can be achieved. Combined with the use of constructs containing regulatory elements that drive expression in not only a temporally way but also in an area-, layer-, and neuronal cell population-specific manner will greatly enhance our knowledge of the functions of particular susceptibility genes playing a role in brain areaspecific cognitive deficits associated with neurodevelopmental disorders.

\section{REFERENCES}

Alvarez-Maya, I., Navarro-Quiroga, I., Meraz-Rios, M. A., Aceves, J., and Martinez-Fong, D. (2001). In vivo gene transfer to dopamine neurons of rat substantia nigra via the highaffinity neurotensin receptor. Mol. Med. 7, 186-192.

Bai, J., Ramos, R. L., Paramasivam, M., Siddiqi, F., Ackman, J. B., and LoTurco, J. J. (2008). The role of DCX and LIS1 in migration through the lateral cortical stream of developing forebrain. Dev. Neurosci. 30, 144-156.

Bonnin, A., Torii, M., Wang, L., Rakic, P., and Levitt, P. (2007). Serotonin modulates the response of embryonic thalamocortical axons to netrin-1. Nat. Neurosci. 10, 588-597.

Borrell, V., Yoshimura, Y., and Callaway, E. M. (2005). Targeted gene delivery to telencephalic inhibitory neurons by directional in utero electroporation. J. Neurosci. Methods 143, 151-158.

Cetin, A., Komai, S., Eliava, M., Seeburg, P. H., and Osten, P. (2006). Stereotaxic gene delivery in the rodent brain. Nat. Protoc. 1, 3166-3173.

Davidson, B. L., and Breakefield, X. O. (2003). Viral vectors for gene delivery to the nervous system. Nat. Rev. 4, 353-364.

Elias, G. M., Elias, L. A., Apostolides, P. F., Kriegstein, A. R., and Nicoll, R. A. (2008). Differential trafficking of AMPA and NMDA receptors by SAP102 and PSD-95 underlies synapse development. Proc. Natl. Acad. Sci. U.S.A. 105, 20953-20958.

Fazzari, P., Paternain, A. V., Valiente, M., Pla, R., Lujan, R., Lloyd, K., Lerma, J., Marin, O., and Rico, B. (2010). Control of cortical GABA circuitry development by Nrg1 and ErbB4 signalling. Nature 464, 1376-1380.

Fukuchi-Shimogori, T., and Grove, E. A. (2001). Neocortex patterning by the secreted signaling molecule FGF8. Science 294, 1071-1074.

Gal, J. S., Morozov, Y. M., Ayoub, A. E., Chatterjee, M., Rakic, P., and Haydar, T. F. (2006). Molecular and morphological heterogeneity of neural precursors in the mouse neocortical proliferative zones. J. Neurosci. 26, 1045-1056.
Garcia-Frigola, C., Carreres, M. I., Vegar, C., and Herrera, E. (2007). Gene delivery into mouse retinal ganglion cells by in utero electroporation. BMC Dev. Biol. 7, 103. doi:10.1186/1471-213X-7-103

Gelman, D. M., Martini, F. J., NobregaPereira, S., Pierani, A., Kessaris, N., and Marin, O. (2009). The embryonic preoptic area is a novel source of cortical GABAergic interneurons. J. Neurosci. 29, 9380-9389.

Gould, T. D., and Gottesman, I. I. (2006). Psychiatric endophenotypes and the development of valid animal models. Genes Brain Behav. 5, 113-119.

Inoue, T., and Krumlauf, R. (2001). An impulse to the brain - using in vivo electroporation. $\mathrm{Nat}$. $\mathrm{Neu}$ rosci. 4(Suppl.), 1156-1158.

Kamiya, A. (2009). Animal models for schizophrenia via in utero gene transfer: understanding roles for genetic susceptibility factors in brain development. Prog. Brain Res. 179, 9-15.

Kawauchi, D., Taniguchi, H., Watanabe, H., Saito, T., and Murakami, F. (2006). Direct visualization of nucleogenesis by precerebellar neurons: involvement of ventricledirected, radial fibre-associated migration. Development 133, 1113-1123.

Kawauchi, T., Chihama, K., Nabeshima, Y., and Hoshino, M. (2003). The in vivo roles of STEF/Tiam1, Rac1 and JNK in cortical neuronal migration. EMBO J. 22, 4190-4201.

Kellendonk, C., Simpson, E. H., and Kandel, E. R. (2009). Modeling cognitive endophenotypes of schizophrenia in mice. Trends Neurosci. 32, 347-358.

Langevin, L. M., Mattar, P., Scardigli, R., Roussigne, M., Logan, C., Blader, P., and Schuurmans, C. (2007). Validating in utero electroporation for the rapid analysis of gene regulatory elements in the murine telencephalon. Dev. Dyn. 236, 1273-1286.

Lewis, T. L. Jr., Mao, T., Svoboda, K., and Arnold, D. B. (2009). Myosindependent targeting of transmembrane proteins to neuronal dendrites. Nat. Neurosci. 12, 568-576.

\section{ACKNOWLEDGMENTS}

We thank members of the Martens Lab for helpful comments on the manuscript. The first author is obliged to the lab of Dr. M. Donoghue and Prof. Dr. P. Rakic, Yale University, School of Medicine, New Haven, USA for learning the technique of in utero electroporation-mediated gene transfer. Also, we would like to apologize for those primary works not referenced here due to space limitations.

Lopez-Bendito, G., Sanchez-Alcaniz, J. A., Pla, R., Borrell, V., Pico, E., Valdeolmillos, M., and Marin, O. (2008) Chemokine signaling controls intracortical migration and final distribution of GABAergic interneurons. $J$. Neurosci. 28, 1613-1624.

LoTurco, J., Manent, J. B., and Sidiqi, F. (2009). New and improved tools for in utero electroporation studies of developing cerebral cortex. Cereb. Cortex 19(Suppl. 1), i120-i125.

Manent, J. B., Wang, Y., Chang, Y., Paramasivam, M., and LoTurco, J. J. (2009). Dcx reexpression reduces subcortical band heterotopia and seizure threshold in an animal model of neuronal migration disorder. Nat. Med. 15, 84-90.

Matsuda, T., and Cepko, C. L. (2007) Controlled expression of transgenes introduced by in vivo electroporation. Proc. Natl. Acad. Sci. U.S.A. 104 1027-1032.

Miyagi, S., Saito, T., Mizutani, K., Masuyama, N., Gotoh, Y., Iwama, A., Nakauchi, H., Masui, S., Niwa, H., Nishimoto, M., Muramatsu, M., and Okuda, A. (2004). The Sox-2 regulatory regions display their activities in two distinct types of multipotent stem cells. Mol. Cell. Biol. 24, 4207-4220.

Mizutani, K., and Saito, T. (2005). Progenitors resume generating neurons after temporary inhibition of neurogenesis by Notch activation in the mammalian cerebral cortex. Development 132, 1295-1304.

Nakahira, E., and Yuasa, S. (2005). Neuronal generation, migration, and differentiation in the mouse hippocampal primoridium as revealed by enhanced green fluorescent protein gene transfer by means of in utero electroporation. J. Comp. Neurol. 483, 329-340.

Navarro-Quiroga, I., Chittajallu, R., Gallo, V., and Haydar, T. F. (2007). Long-term, selective gene expression in developing and adult hippocampal pyramidal neurons using focal in utero electroporation. J. Neurosci. 27, 5007-5011.

Niwa, M., Kamiya, A., Murai, R., Kubo, K., Gruber, A. J., Tomita, K., Lu, L., Tomisato, S., Jaaro-Peled, H., Seshadri, S., Hiyama, H., Huang, B., Kohda, K., Noda, Y., O’Donnell,
P., Nakajima, K., Sawa, A., and Nabeshima, T. (2010). Knockdown of DISC1 by in utero gene transfer disturbs postnatal dopaminergic maturation in the frontal cortex and leads to adult behavioral deficits. Neuron 65, 480-489.

North, H. A., Zhao, X., Kolk, S. M., Clifford, M. A., Ziskind, D. M., and Donoghue, M. J. (2009). Promotion of proliferation in the developing cerebral cortex by EphA4 forward signaling. Development 136, 2467-2476.

Petreanu, L., Huber, D., Sobczyk, A., and Svoboda, K. (2007). Channelrhodopsin-2-assisted circuit mapping of long-range callosal projections. Nat. Neurosci. 10, 663-668.

Petros, T. J., Rebsam, A., and Mason, C. A. (2009a). In utero and ex vivo electroporation for gene expression in mouse retinal ganglion cells. J. Vis. Exp. 31, 1-6.

Petros, T. J., Shrestha, B. R., and Mason, C. (2009b). Specificity and sufficiency of EphB1 in driving the ipsilateral retinal projection. J. Neurosci. 29, 3463-3474.

Punzo, C., and Cepko, C. L. (2008). Ultrasound-guided in utero injections allow studies of the development and function of the eye. Dev Dyn. 237, 1034-1042.

Punzo, C., Kornacker, K., and Cepko, C. L. (2009). Stimulation of the insulin/mTOR pathway delays cone death in a mouse model of retinitis pigmentosa. Nat. Neurosci. 12, 44-52.

Remedios, R., Huilgol, D., Saha, B., Hari, P., Bhatnagar, L., Kowalczyk, T., Hevner, R. F., Suda, Y., Aizawa, S., Ohshima, T., Stoykova, A., and Tole, S. (2007). A stream of cells migrating from the caudal telencephalon reveals a link between the amygdala and neocortex. Nat. Neurosci. $10,1141-1150$.

Saito, T., and Nakatsuji, N. (2001). Efficient gene transfer into the embryonic mouse brain using in vivo electroporation. Dev. Biol. 240, 237-246.

Soma, M., Aizawa, H., Ito, Y., Maekawa, M., Osumi, N., Nakahira, E., Okamoto, H., Tanaka, K., and Yuasa, S. (2009). Development of the mouse 
amygdala as revealed by enhanced green fluorescent protein gene transfer by means of in utero electroporation. J. Comp. Neurol. 513, 113-128.

Tsai, J. W., Bremner, K. H., and Vallee, R. B. (2007). Dual subcellular roles for LIS1 and dynein in radial neuronal migration in live brain tissue. Nat. Neurosci. 10, 970-979.

Vreugdenhil, E., Kolk, S. M., Boekhoorn, K., Fitzsimons, C. P., Schaaf, M., Schouten, T., Sarabdjitsingh, A., Sibug, R., and Lucassen, P. J. (2007). Doublecortin-like, a microtubuleassociated protein expressed in radial glia, is crucial for neuronal precursor division and radial process stability. Eur. J. Neurosci. 25, 635-648.
Wang, C. L., Zhang, L., Zhou, Y., Zhou, J., Yang, X. J., Duan, S. M., Xiong, Z. Q., and Ding, Y. Q. (2007a). Activitydependent development of callosal projections in the somatosensory cortex. J. Neurosci. 27, 11334-11342.

Wang, X., Qiu, R., Tsark, W., and Lu, Q. (2007b). Rapid promoter analysis in developing mouse brain and genetic labeling of young neurons by doublecortin-DsRed-express. $J$. Neurosci. Res. 85, 3567-3573.

Willett, R. T., and Greene, L. A. (2011). Gata2 is required for migration and differentiation of retinorecipient neurons in the superior colliculus. J. Neurosci. 31, 4444-4455.

Young-Pearse, T. L., Bai, J., Chang, R., Zheng, J. B., LoTurco, J. J., and
Selkoe, D. J. (2007). A critical function for beta-amyloid precursor protein in neuronal migration revealed by in utero RNA interference. $J$. Neurosci. 27, 14459-14469.

Zhang, F., Gradinaru, V., Adamantidis, A. R., Durand, R., Airan, R. D., de Lecea, L., and Deisseroth, K. (2010). Optogenetic interrogation of neural circuits: technology for probing mammalian brain structures. Nat. Protoc. 5, 439-456.

Conflict of Interest Statement: The authors declare that the research was conducted in the absence of any commercial or financial relationships that could be construed as a potential conflict of interest.
Received: 08 July 2011; accepted: 11 October 2011; published online: 01 November 2011.

Citation: Kolk SM, de Mooij-Malsen AJ and Martens GJM (2011) Spatiotemporal molecular approach of in utero electroporation to functionally decipher endophenotypes in neurodevelopmental disorders. Front. Mol. Neurosci. 4:37. doi: 10.3389/fnmol.2011.00037

Copyright $\odot 2011$ Kolk, de Mooij-Malsen and Martens. This is an open-access article subject to a non-exclusive license between the authors and Frontiers Media $S A$, which permits use, distribution and reproduction in other forums, provided the original authors and source are credited and other Frontiers conditions are complied with. 